\title{
Nanobiotecnología: una descripción general
}

\author{
Flavio F. Contreras-Torres* y Elena V. Basiuk
}

Centro de Ciencias Aplicadas y Desarrollo Tecnológico, Universidad Nacional Autónoma de México, Circuito Exterior, Ciudad Universitaria 045 I0, México D.F., México

*Autor para correspondencia: flavioc@nucleares.unam.mx.

\begin{abstract}
Resumen
La nanobiotecnología representa la faceta más intrigante que pudo alguna vez haber alcanzado en sí la tecnología al servicio del ser humano. Al tomar ventaja de las características adquiridas por los organismos vivos en el curso de su evolución, más las propiedades únicas que los materiales presentan a nanoescala, esta nueva tecnología nos promete el desarrollo de dispositivos con una complejidad nunca antes imaginada. Por ejemplo, biotecnología de nanoestructuras basadas en péptidos, proteínas e incluso virus y bacterias, todas ellas combinadas con nanoestructuras inorgánicas, dando lugar a la formación de nuevos materiales bionanohíbridos con vastas aplicaciones en el área de las ciencias de la vida y de los materiales. En una forma general, la nanobiotecnología abarca el estudio, diseño y creación de líneas de conexión entre la biología estructural y la nanotecnología molecular, recalcando que su desarrollo no habría sido posible sin un esfuerzo conjunto y multidiciplinario de todas las áreas de la ciencia. En esta revisión se presenta un fascinante universo de nuevos materiales en la escala $1 \times 10^{-09}$ metros cuyas aplicaciones pueden hacernos reflexionar hacia donde se dirigen nuestros días futuros. Sin embargo, dada la vasta cantidad de artículos científicos publicados en el área, la presente revisión solamente se ha enfocado en el uso de nanotubos de carbono para el desarrollo de nanobiodispositivos, y se apoya en aproximadamente un centenar de referencias científicas.
\end{abstract}

Palabras clave: Biotecnología, nanomateriales, materiales biohíbridos, nanotubos de carbono.

\begin{abstract}
Nano-biotechnology is the most interesting accomplishment of present-day technology at the disposal of human beings. By taking advantage of characteristics acquired by living organisms in the course of evolution plus the unique properties of materials at nano-scale, this new technology anticipates the development of devices with a complexity never imagined before. For instance, the biotechnology of nano-structures based on peptides or even on viruses and bacteria, all of them combined in inorganic nano-structures, thereby giving rise to new nano-bio-hybrid materials with ample application in the area of the sciences of life and that of materials themselves. In broad terms, nano-biotechnology includes the study, design and creation of links between structural biology and molecular nano-technology. Its development was made possible by a joint and multidisciplinary effort in all scientific fields. This overview describes a fascinating universe at a scale of I ? 10-09 meter, the application of which should makes us think about where our future lives are heading. Nevertheless, given the enormous amount of scientific papers published on the field, the present overview focuses on the use of carbon nano-tubes for the development of nano-biodevices and draws on some hundred scientific references
\end{abstract}

Key words: Biotechnology, nano-materials, biohybrid materials, carbon nano-tubes.

La Granja 9(I): 3-15. 2009.

(C) 2009, Universidad Politécnica Salesiana, Ecuador. 


\section{Antecedentes}

El desarrollo de nuevos materiales no ha estado siempre en comunión con las políticas ecológicas o en afinidad con las medidas de salubridad y de subsistencia como pretende ahora estar la nanotecnología. En una era donde la seguridad económica, ambiental y social es crucial para un próspero desarrollo, esta tecnología, generalmente definida como el control sobre los materiales (léase también átomos y moléculas) en una forma precisa en la escala de $\mathrm{I}-100 \mathrm{~nm}$, aparece como el puntal asociado con dicho crecimiento.

Siendo de reconocimiento general que el primer gran impacto de una nueva tecnología radicará siempre en el aspecto económico, y por ello consecuentemente desplazará a las sociedades hacia un desarrollo diferente, es necesario tener desde ahora una comprensión mínima de lo que es y pretende hacer la nanotecnología en los años siguientes. Según el criterio personal de varios expertos, la nanotecnología tiene la capacidad de cambiar al mundo, como económicamente ya ha empezado hacerlo. Por ejemplo, de acuerdo a fuentes industriales (Mashelkar, 2006) fue estimado que para el año 2008 habrían sido empleados alrededor de USD 3.210 millones en gastos de investigación y desarrollo en nanociencias y cuyo liderazgo, básicamente, estaría a cargo de EE.UU. (65\%), seguido por la Unión Europea (20\%) y el Japón (10\%). Además, de acuerdo a estimados del US $\mathrm{Na}$ tional Science Foundation (Nanobusiness Alliance, 200I) se pronostica que para el año 2015 el mercado de productos a nano escala alcanzará la cifra record de USD I trillion per annum. Bajo este contexto y cifras en la mano, no es posible dejar de pensar en cuáles cambios venideros sobre nuestras formas de vida y corrientes del pensamiento posmoderno habrán de suceder. Actualmente ya existen coyunturas desde el campo legal hasta el filosófico sobre el desarrollo, uso, producción y manipulación de nanomateriales, $y$ dónde son muy discutidas las evaluaciones de toxicidad de los nanomateriales (Hussain \& Terrones, 2009). Un ejemplo especial es el debate médico-psíquico-legal generado por las investigaciones en neuronanociencias donde ha quedado planteado el problema acerca de si el tratamiento con nanomedicina podría o no afectar a nuestro sentido común, en el sentido de dar atribuciones diferentes a los estados mentales, como la responsabilidad, incluyendo la responsabilidad criminal (Sifferd, 2008).

Por otro lado, los términos nanobiotecnología y bionanotecnología, usados intercambiablemente, son el conjunto de las herramientas y procesos emplea- dos en la nano/microfabricación de nuevos dispositivos para estudiar y manipular biosistemas. Heredera de la tecnología del manejo del ADN recombinante, hoy en día la nanobiotecnología es una disciplina en franco desarrollo, y se dice que constituye un campo emergente dada la necesidad de superar no solamente los retos biotecnológicos actuales, sino también las barreras coyunturales que mantienen -por muy poco tiempo más- separadas a las diversas disciplinas científicas. Posiblemente, en un futuro cercano, la investigación transdisciplinaria sea la que conduzca el desarrollo de nuevas nanobioestructuras moleculares mismas que en forma de productos finales de consumo podrán beneficiar a las próximas generaciones con nanotecnología en sensores químicos (Sun, 2007), biosensores (Teles, 2008), transportadores moleculares de fármacos (Langer, 2004; Beaux II, 2008; Dinu, 2009), tejidos artificiales (Mooney, 1999) y neuronanoprotectores cerebrales (Linazasoro, 2008).

Siendo la compatibilidad entre materiales a diversas escalas el punto clave dentro del proceso de la nanomanufactura, el diseño a estas dimensiones requiere de una tecnología altamente versátil que se pueda acomodar al amplio rango de las propiedades, sean estas químicas o físicas. Los estudios científicos más actuales (Mitragotri, 2009) revelan que en la misma forma como las propiedades químicas (e.g. reconocimiento molecular) trabajan en beneficio del diseño de nuevos materiales, también los atributos físicos (e.g, tamaño, forma y propiedades mecánicas) deben ser explotados con el fin de establecer bases más sólidas al momento de conceptualizar el diseño de bionanomateriales.

Los primeros beneficios de dichas estrategias ya son observables. Por ejemplo, una combinación de forma, tamaño y química de superficies ha sido usada para focalizar partículas al endotelium vascular como una aplicación al suministro de fármacos (Muro, 2008). De igual manera, una combinación de forma y flexibilidad mecánica ha sido usada para focalizar más eficientemente fármacos en tumores (Gen, 2007). En este último caso, la flexibilidad mecánica de las nanopartículas les permite mantenerse dentro de la circulación sanguínea por mucho más tiempo. Tal extraordinaria correlación, no obstante, es solamente consistente con la noción que el reconocimiento molecular forma la base de muchas funciones biológicas (Griffith, 2006), es decir, que la geometría local del microambiente también es un factor preponderante que influye sobre el diseño y control de los nuevos materiales, tal como ha sido estudiado en la ingeniería de tejidos a través de la muerte celular (Chen, 
1997). Estos primeros ejemplos en nanotecnología no solamente están intencionados con promover nuevas iniciativas de desarrollo en el sector de la salud, sino en los sectores agrícola y energético. Por ejemplo, se destaca el reciente uso de la nanosílica amorfa como un biopesticida de alto impacto (Barik, 2008) y el empleo de nanotubos de carbono para el almacenamiento de combustibles ecológicos (Kong, 200I). Bajo este contexto, la nanotecnología es una amplia área interdisciplinaria que compromete la investigación en cuatro áreas específicas: I) nanomedicina, 2) nanofabricación, 3) nanometrología y 4) nanomateriales.

En materia de desarrollo, la historia de la nanotecnología puede ser comprendida desde dos posiciones, las que están relacionadas ya sea con el diseño artificial de nuevos materiales inorgánicos (i.e., edad posindustrial) o con los procesos naturales accidentales ocurrentes en los compuestos orgánicos como las proteínas y el ADN (i.e., origen de la vida). Sin embargo, dada la notable reputación del científico Richard Feymann, quien estuvo dedicado a elaborar teorías, principalmente aplicables dentro de las ingenierías eléctrica, mecánica y de materiales, existe la tendencia general de creer que los inicios nanotecnológicos se remontan al diseño de nanomateriales inorgánicos (e.g. nanotransistores). En contraposición, la ingeniería implícitamente aplicada por las proteínas habría de representar para Erick Drexler (1986) como el ejemplo más claro donde una generación de nanomáquinas trabaja a favor de un proceso. A fin de producir nuevas generaciones en nanomáquinas, el creador del término Nanotecnología Molecular (MNT, por sus siglas en inglés), postuló que las bases ingenieriles del autoensamblaje molecular podrían ser empleadas en el desarrollo de nuevos materiales biohíbridos, mismos que trabajarían en igual forma como lo hacen las proteínas, pero que serían más inteligentes dada su construcción "átomo por átomo" (Drexler, 1991).

La lógica de Drexler, basada en una remembranza con los procesos naturales, dio lugar a la postulación de la Teoría del Grey Goo (ver Joy, 2000), un hipotético escenario del fin del mundo basado en MNT. Hoy en día, las visiones más pragmáticas consideran a la segunda década del presente siglo como el tope en tiempo máximo para desarrollar las técnicas que logren mimetizar a los procesos biológicos. Una posible calendarización en el desarrollo de prototipos comercialmente viables es la siguiente: primera generación o nanoestrucutras pasivas (e.g., nanopartículas) hasta el año 2004; segunda generación o nanoestrucuras activas (e.g., nanotransistores) hasta el año
2009; tercera generación o nanosistemas tridimensionales (e.g., nanocompuestos heterogéneos) hasta el año 20l4; y cuarta generación o nanosistemas moleculares inteligentes capaces de auto ensamblarse (ca. año 2025).

Entre los artículos actualmente fabricados con materiales nanoestructurados de primera generación que ya se encuentran presentes en el mercado se mencionan: partes para bolas de tenis, golf o boliche, neumáticos de alto rendimiento, telas antimanchas, filtros/membranas para agua, cosméticos, entre otros. Además, productos derivados para la industria militar, no conocidos dada su naturaleza confidencial, y productos de uso civil empleados en tareas específicas como la investigación, que representan en su conjunto a un sector social dedicado al desarrollo nanotecnológico. Bajo este contexto poblacional (i.e., sociedades civil, militar y científica) el comercio de los nanomateriales, poco a poco, llegará a involucrar al grueso total de la población mundial, éstos no solamente como consumidores finales, sino también como empleados manufactureros. De ahí que el tema de la biocompatibilidad de los nanomateriales, actualmente en fase de estudio, representa uno de los tópicos más controversiales al hablar de nanobiotecnología. Y es que después de casi dos décadas de haber sido descubierto uno de los principales nanocompuestos, como lo es el fulereno $\mathrm{C}_{60}$ (Kroto, 1985), aún se desconoce su verdadera incidencia en la salud humana.

Entre los riesgos e implicaciones del uso y manipulación de artículos manufacturados a partir de nanomateriales se destacan: I) que la promesa de reducir costos por energía no necesariamente se cumpliría si se toma en cuenta tanto a los costos de producción como a los de investigación, uso y desecho; 2) que la manipulación de estos artefactos no solamente podría comprometer a la genética del ser humano, sino también a la de virus y bacterias con consecuencias impredecibles; 3 ) que de cumplirse todas las expectativas esperadas en dichos materiales éstas no necesariamente se vincularían con los estratos sociales bajos; 4) que las operaciones con dichos materiales en el campo militar podrían violentar los derechos civiles al transformar la naturaleza de las guerras; 5) que el actual avance de la bionanotecnología podría resultar dentro de un mediano a largo plazos en una transformación total de la sociedad actual (Delgado, 2008). Mientras que, por una parte, la Administración Federal de Alimentos y Fármacos de Estados Unidos (FDA, por sus siglas en inglés) no ha advertido aún la necesidad argumentando que todavía no existe evidencia científica que así lo demuestre de 


\section{Contreras Torres y Basiuk}

implementar normativas tan simples como el etiquetar con anuncios especiales tanto a los medicamentos como a los cosméticos u otros productos de uso humano o animal elaborados basados en nanomateriales (Eschenbach, 2007), por otro lado numerosas investigaciones siguen enfocadas por conocer la conducta de los nanomateriales al contacto con los sistemas biológicos (Hussein, 2009). En ésta, tanto las reflexiones éticas como las morales sobre el uso y la producción de los nanomateriales pueden verse fácilmente empañadas con tintes opuestos, entendido como el uso pro bien (o pro mal) de dichos materiales.

En este punto, es difícil dejar de lado la significancia que tuvieron los debates en biotecnología y bioética para entender la demanda por la nanoética. En Europa Occidental, por ejemplo, el tema de la biotecnología agrícola resultó ser un capítulo tan controversial que fue discutido desde puntos de vista extremadamente sutiles como las afirmaciones en los dos sentidos siguientes: I) la ausencia de evidencia de riesgo, y 2) la evidencia de ausencia de riesgo. Dado que ambos sentidos gozan de una verdad (aunque sea parcial), el punto clave del desarrollo nanotecnológico de un "X" todavía no existente debería estar enfocado hacia el suscrito ético, desde sus primeras etapas (Strand \& Nydal, 2008).

\section{Microscopía de fuerza atómica: los ojos de un mundo nanométrico}

Desde su invención en 1986 (Binnig, 1986), el microscopio de fuerza atómica (AFM, por sus siglas en inglés) se ha convertido en la herramienta más importante para el estudio a nivel atómico de una amplia variedad de superficies o para la investigación de las propiedades mecánicas (e.g., fricción y rigidez), propiedades eléctricas (e.g., potencial superficial, polarización eléctrica, carga en la superficie), propiedades electromecánicas (e.g., piezoelectricidad) y propiedades magnéticas relacionadas. Aunque la microscopía por AFM es una técnica más entre la gran familia de microscopías de barrido por sonda, y entre las cuales se encuentra la microscopía de tunelaje (Binning, 1982), su capacidad para estudiar no solamente superficies inorgánicas, sino también moléculas orgánicas (incluyendo ADN y células) adheridas a las superficies ha propiciado que la comunidad científica llame a esta técnica como el ojo de la nanotecnología. Debido a la extensa bibliografía enfocada en la explicación de los fundamentos físicos del AFM, esta revisión no pretende volver a mencionarlas (el lector no obstante puede referirse a Ralston, 2005). En su lugar, nom- bramos brevemente aquellos principios básicos, físicos e instrumentales, que permiten comprender de una forma sucinta cualquier experimento relacionado con esta técnica.

En primer lugar, la instrumentación de un microscopio de AFM está compuesta por un piezoeléctrico, el cual básicamente es una cerámica que se expande y contrae al estar expuesto a un voltaje. Dicha expansión (o contracción) está en el orden de los nanómetros $(\mathrm{nm})$ y se dirige sobre un eje $z$. Sobre el piezo se monta una sonda rectangular que en su extremo saliente tiene depositado cúmulos de átomos de silicio ( $\mathrm{Si})$ o, en su caso, de nitruro de silicio $\left(\mathrm{Si}_{3} \mathrm{~N}_{4}\right)$ en una forma piramidal (Figura I). Los átomos de la punta interactuarán con los átomos presentes en la superficie a través de fuerzas (i.e., atracciones y/o repulsiones de sus respectivas nubes electrónicas) las que provocarán una deflexión de la sonda. Por lo anterior, la sensibilidad de la sonda de AFM, conocida también como la constante $(k)$ de resorte del material, es un factor muy importante para determinar las propiedades de las muestras en estudio; su respuesta al contacto con una determinada superficie es un reflejo directo de las interacciones intra o intermoleculares presentes.

El escaneado (barrido) del material se desarrolla por servomotores que se encargan de exponer la sonda de AFM al contacto con una serie definida de puntos $(x, y)$ de la superficie. La selección correcta de una sonda de AFM es extremadamente importante para obtener resultados que reflejen una alta calidad en la señal obtenida (i.e., imágenes topográficas claras). Cada sonda de AFM tiene su frecuencia de resonancia característica (entre 100 y $500 \mathrm{kHz}$ ) misma que es determinada in situ una vez montada la sonda sobre el piezo.

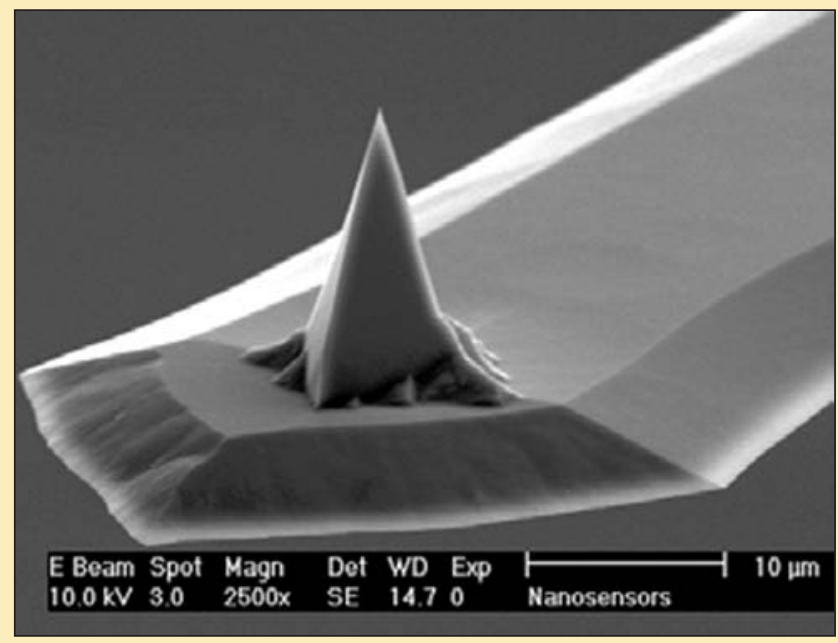

Figura I. Sonda de AFM empleada para el barrido de una superficie. Fuente:Agilent Technologies. 
La señal principal de una medición por AFM, que puede ser una imagen topográfica, una de fase, $O$ una de amplitud, entre las más importantes, es construida como un tensor de segundo rango dado al acoplamiento entre los vectores de la señal de excitación (fuerzas; medida en $z$ ) y la señal de respuesta (desplazamiento; medida por los puntos $x, y$ ). Cabe aclarar que el orden de las fuerzas medidas es aproximadamente del orden de picoNewtons ( $\mathrm{pN}$ ). Finalmente, la dependencia entre la magnitud de dichas fuerzas con respecto a la distancia de la sonda a la muestra da lugar a tres regímenes de trabajo: no contacto, contacto y contacto intermitente (Figura 2). Una típica fuerza presente en cualquier medición es la fuerza de capilaridad ( que actúa en el modo de contacto); por otra parte, las fuerzas de van der Waals (vdW) aparecen por la interacción a grandes distancias entre el sustrato y la sonda de medición (presentes en el modo de contacto intermitente). Otras fuerzas presentes son la fuerza electrostática y la magnética (presentes en el modo de no contacto). Normalmente, la microscopía de fuerzas en muestras biológicas se realiza en el modo de contacto intermitente (Dulinska, 2006; Kienberger, 2006) esto, entre otras razones, debido a la necesidad de reducir al máximo posible un daño prematuro a la membrana de la muestra e.g. virus (Ross, 2009).

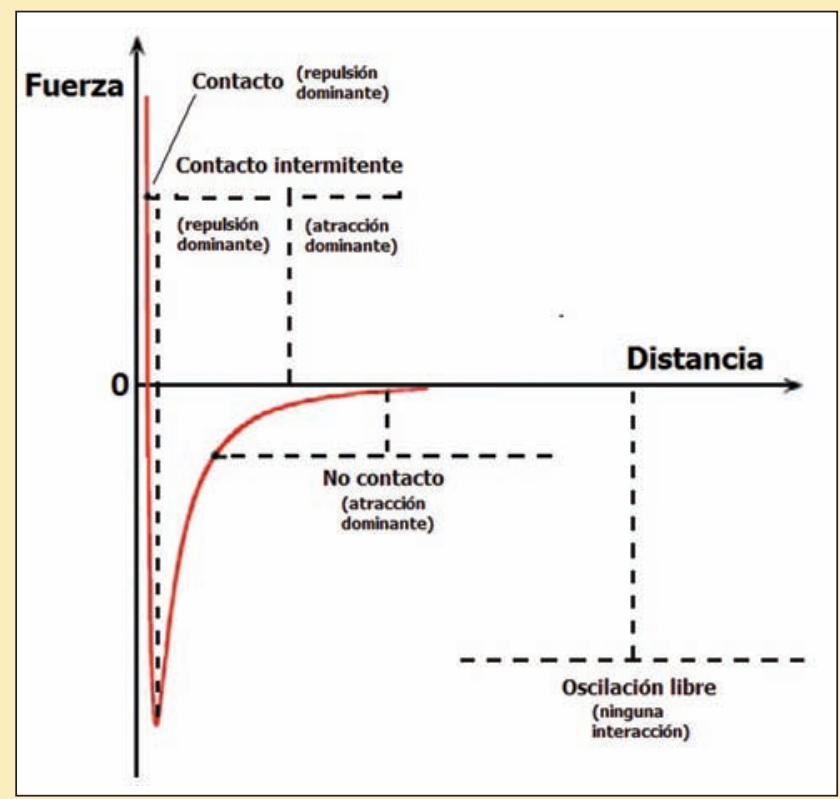

Figura 2. Curva típica de fuerzas vs. distancia donde se indica los tres regímenes de trabajo en AFM (contacto, contacto intermitente y no contacto). Fuente Flavio Contreras-Torres

\section{Nanocompuestos de carbono en aplicaciones biotecnológicas}

Basados en sus exclusivas propiedades fotofísicas y electróquímicas, las nanoestructuras inorgánicas más importantes son los fulerenos y los nanotubos de carbono. Dichos nanomateriales de carbono actualmente son ampliamente empleados en el desarrollo de nuevas arquitecturas biohíbridas bi y tridimensionales con posibles futuros usos en biotecnología médica. La presente revisión se ha enfocado solamente al uso de nanotubos de carbono y sus aplicaciones biotecnológicas (Harutyunyan, 2002) con un especial interés en el desarrollo de biosensores.

Los nanotubos de carbono (CNTs, por sus siglas en inglés) pueden ser visualizados como una hoja de grafito enrollada con una helicidad particular, que puede ser expresada teóricamente a través de un vector quiral $\underline{R}_{\mathrm{nm}}$ (Figura 3). Dependiendo del giro del vector $R_{n m}=n \cdot a_{1}+m \cdot a_{2}$, los nanotubos pueden tener las orientaciones armchair $(n=m)$ y zigzag $(m=0)$. Sin embargo, también se pueden presentar un vasto número de orientaciones medias llamadas quirales $(n \neq$ $m$ ).Así, el comportamiento metálico o semiconductor de los CNTs es inferido por el carácter helicolidal de los tubos. Por ejemplo, si un nanotubo presenta un giro $n-m=$ ?3? (i.e., módulo tres) se considera que es metálico, de otra forma será semiconductor. El diáme-

a
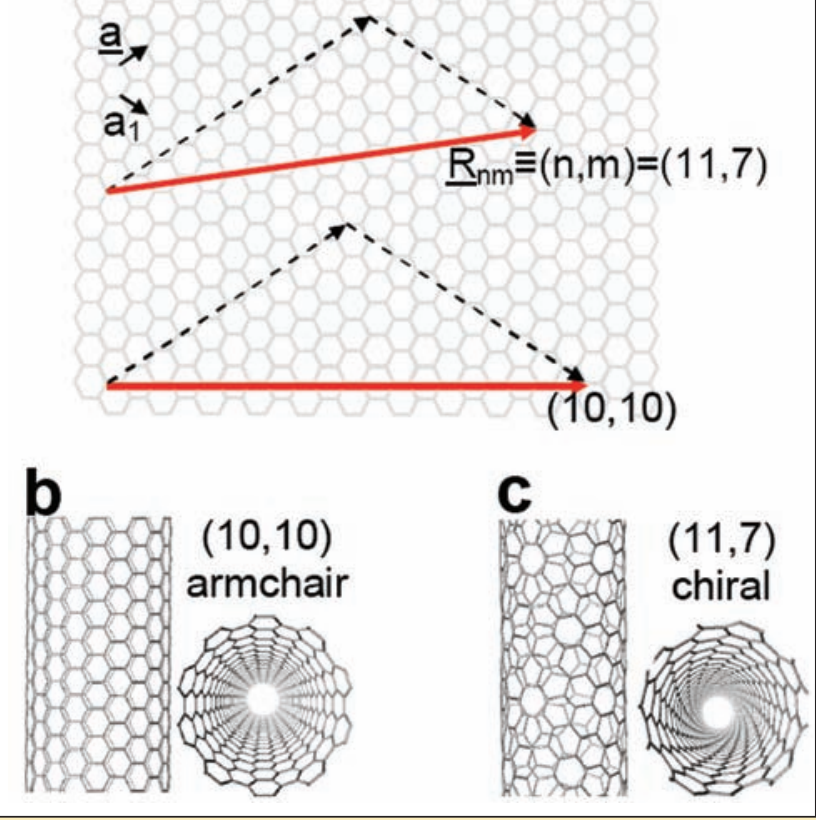

Figura 3. Nanotubos de carbono: (a) descripción de su quiralidad por medio del vector Rnm a través del enrollamiento de una hoja de grafeno (b) nanotubo armchair;y (c) nanotubo quiral. Fuente Flavio Contreras-Torres 


\section{Contreras Torres y Basiuk}

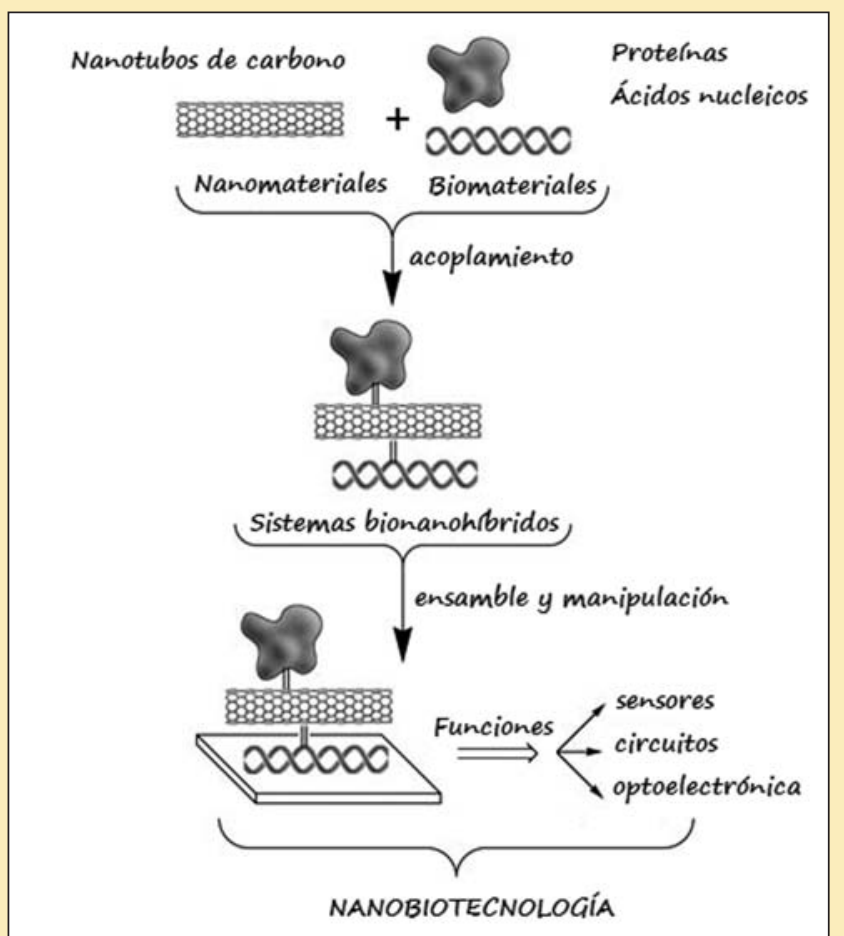

Figura 4. Representación conceptual sobre la generación de sistemas nanobiohíbridos y sus diferentes funciones nanobiotecnológicas que pueden alcanzar. (Adaptado al español—sin permiso-desde Katz, 2004. (C) 2004 Willey-VCH Verlag GmbH \& Co. KGaA, Weinheim).

tro de un CNT no es constante y depende básicamente si se corresponde a un solo tubo (Single-walled carbon nanotube, SWCNT) o a un aglomerado concéntrico de tubos (Multi-walled carbon nanotubes, MWCNT). Los nanotubos de capa simple, SWCNT (CNTs para acortar), pueden aproximadamente variar entre los 0,2-2 $\mathrm{nm}$ y sus longitudes pueden llegar hasta los centímetros. Es necesario recordar que no existe un descubridor formal de estos nanocompuestos de carbono (Monthioux, 2006) pero se le atribuye el reconocimiento al profesor Sumio ljima (ljima, 199l) por ser la primera persona en haberlos reportado como tales $y$, sobre todo, por haberlos bautizado los con un nombre muy característico.

En esta forma el acoplamiento entre nanomateriales (e.g., nanotubos de carbono, fulerenos, nanopartículas metálicas) con biomateriales (e.g., proteínas/enzimas, antígenos/anticuerpos o ADN) provee de nuevas rutas para formar nanomateriales biohíbridos que podrán combinar las propiedades físicas de los nanomateriales (e.g., conductividad o semiconductivad de los CNTs) con las propiedades bioquímicas de los biomateriales (e.g., reconocimiento o propiedades catalíticas). Se muestra (Figura 4) una representación conceptual (Katz, 2004) sobre la genera- ción de nanobiomateriales y sus principales aplicaciones como sensores, circuitos y finalmente elementos electrónicos y optoelectrónicos.

\section{I Inmunosensores}

Los recientes desarrollos en nanotecnología han allanado el camino para un gran número de nuevos materiales y dispositivos con propiedades únicas, muchos de los cuales se espera logren alcanzar funciones útiles en el campo de la biotecnología. En términos generales los sensores pueden ser clasificados en sensores químicos y biosensores, diferenciándose por el método electroquímico empleado para el proceso, Balasubramanian (2006), Vestergaard (2007) y Yogeswaran (2008). La selección y desarrollo de un Material de Detección Activo (MDA) es una área en franco desarrollo, aunque su base consiste siempre en actuar como un catalizador para la oxidación o reducción de un analito particular.

En particular, los Inmunosensores (IS), un tipo especial de biosensores, pueden ser definidos como artefactos analíticos compactos constituidos por anticuerpos o antígenos (o sus fragmentos) integrados a un transductor fisicoquímico (Veetil, 2007). La importancia de los inmunosensores, en medicina por ejemplo, radica en el hecho de que generan una señal selectiva y específica dada una reacción particular de un antígeno $o$ anticuerpo empleado con terceras sustancias. A partir de esta particularidad se pueden considerar a los IS como detectores de eventos moleculares específicos; así, sus costos de producción pueden llegar a ser demasiado altos dada la baja sensibilidad que en muchos casos presentan. Amplificar la señal de los IS representa hoy en día un área de estudio y desarrollo muy importante. Para una revisión completa sobre el desarrollo de IS se puede consultar el trabajo de Morgan (2006).

Uno de los métodos más importantes para mejorar la sensibilidad de los IS ha sido reportado mediante la incorporación de nanotubos de carbono dentro de su matriz (Tang, 2004; Li, 2005). Las propiedades de los CNTs les permiten interactuar con algunos compuestos aromáticos orgánicos a través de interacciones electrónicas tipo $\pi-\pi$ y en algunos casos también por interacciones hidrofóbicas (ver e.g., Li, 2004; Liang, 2007; Star, 2003; Zhang, 2003). Entre los MDA empleados en conjunto con CNTs se nombran a poli (rojo neural), Nafion, nanopartículas metálicas, citocromo c (cyt c), hidroxipropil- $\beta$-ciclodextrina, entre otros. Por ejemplo, los compuestos entre CNT y poli (rojo neural) han sido empleados como sensores de NADH, neurotransmisores, o (-)-epiga- 


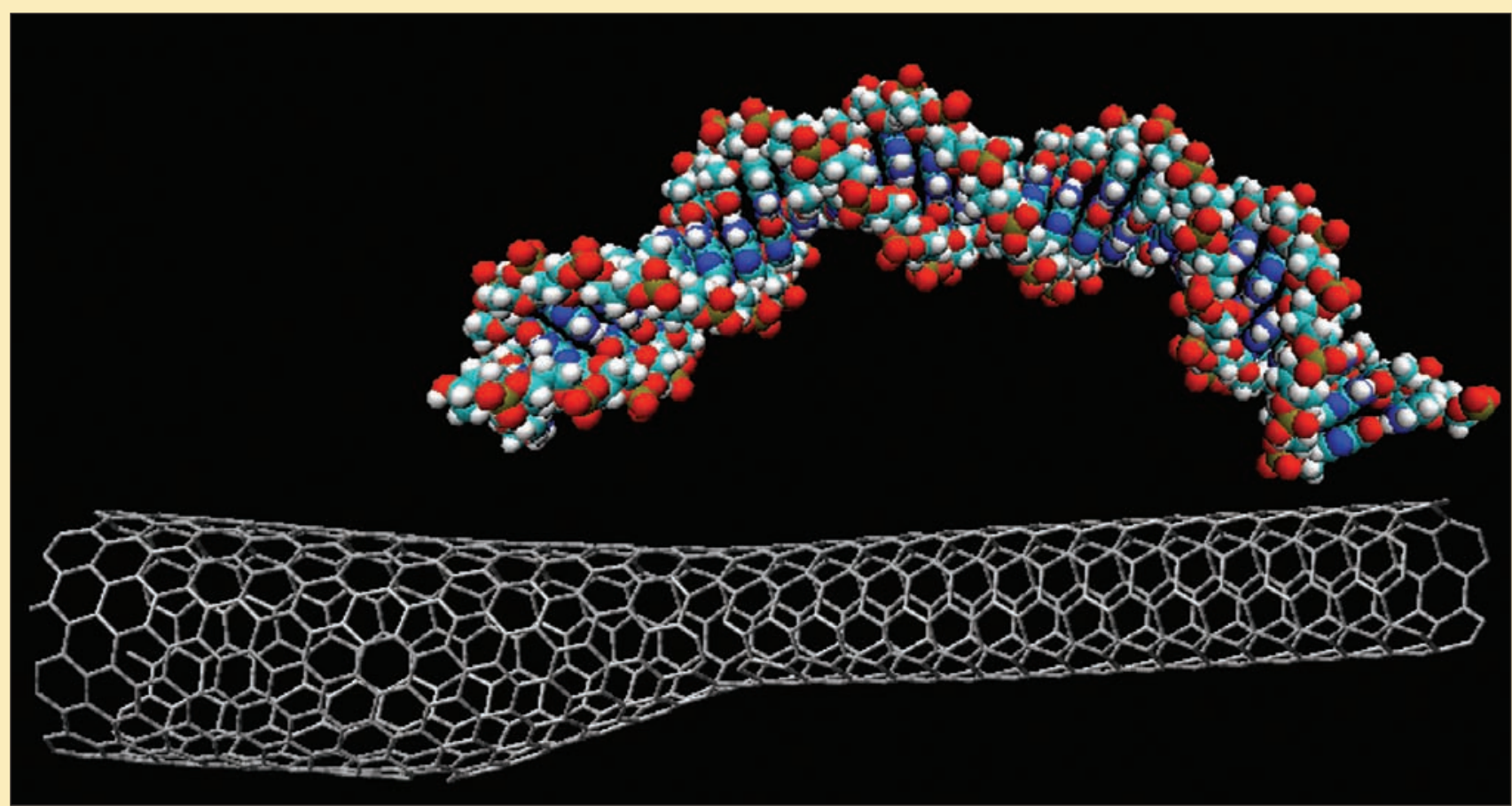

Figura 5. Representación gráfica de una interacción no covalente entre una fragmento de ADN y un modelo de CNT defectuoso por heterouniones. Obsérvese como los extremos de la biomolécula se orientan preferentemente a los defectos del nanotubo (e.g., puntas oxidadas y heterouniones). Fuente: Flavio F. Contreras-Torres.

locatequina galato (Yogeswaran \& Chen, 2007; Yogeswaran, 2007; Zhou, 2005). Similarmente, compuestos formados entre CNTs y cyt $c$ han sido empleados para la detección de ácido ascórbico y L-cisteina (Shie, 2008). Finalmente, los restantes MDA mencionados han sido empleados con CNTs para la detección simultánea de neurotransmisores con ácidos ascórbico y úrico (Yogeswaran, 2007b; Yogeswaran, 2007c; Yogeswaran \& Chen 2008b).

Cabe aclarar el hecho que los CNTs por sí solos son incapaces de reconocer específicamente a biomoléculas, en su lugar es necesario dotarles primeramente de funcionalidades que les permitan adquirir un reconocimiento molecular específico al emitir una señal de transducción; estas metodologías se conocen como funcionalización química.

En términos generales, la funcionalización química (conocida en sus primeros inicios también como derivatización) de los CNTs puede realizarse por las vías covalente y no covalente, aunque para ambas vías la idea básica es inmovilizar biomoléculas sean estas nucleótidos (Contreras-Torres, 2008; Contreras-Torres, 2008b), proteínas (Davis, 1998), péptidos, enzimas, anticuerpos o antígenos (Kam, 2005). Las biomoléculas normalmente permanecerán adheridas ya sea a los extremos de los tubos (puntas) o a sus paredes externas; existe una amplia actividad de investigación enfocada por conocer la reactividad en las paredes internas de los CNTs. Muchas veces el principal objetivo de estas funcionalizaciones es lograr mejorar la solubilidad de los CNTs, de ahí que es necesario reconocer el objetivo principal de la derivatización.

Por ejemplo, el método oxidativo con $\mathrm{HNO}_{3}$ y $\mathrm{H}_{2} \mathrm{SO}_{4}$ es reconocido porque permite incorporar una serie experimentalmente indefinida de grupos oxidados (e.g. $-\mathrm{COOH},-\mathrm{OH}$ ó $\mathrm{C}=\mathrm{O}$ ) en sus puntas y/o cuerpo con lo cual se puede mejorar su pobre hidrofilicidad.Adicionalmente, este tratamiento oxidativo también conlleva a que los nanotubos se acorten y, por lo tanto, a que se reduzca su relación diámetrolongitud muy deseada para otros fines. El paso subsecuente a la incorporación de grupos oxidados en los nanotubos conlleva a una serie de reacciones de amidación y esterificación (Contreras-Torres \& Basiuk, 2007). Teóricamente ha sido demostrado (ContrerasTorres \& Basiuk, 2008c) que esta reacción permitiría poder experimentalmente separar a los nanotubos metálicos (e.g., armchair) de los semiconductores debido a la diferencia calculada para las energías de activación y energías libres derivadas para cada reacción. Los grupos carboxílicos en los CNTs oxidados también pueden ser convertidos en ésteres activos de carbodiimida al tratarlos con $N, N^{\prime}$-diciclohexilcarbodiimida en medios orgánicos (Gooding, 2003).

A fin de conservar las propiedades electrónicas de los CNTs la funcionalización no covalente es pre- 


\section{Contreras $\sim$ Torres y Basiuk}

ferida sobre la covalente, esto debido a que la adición no covalente de terceras moléculas preserva localmente la parcial hibridación $\mathrm{sp}^{2}$ del cuerpo del nanotubo. Entre las aproximaciones no covalentes más comunes se destaca el tratamiento con detergentes 0 surfactantes, como por ejemplo, dodecil sulfato de sodio o Triton-X 100 (Moore, 2003). Por ejemplo, de acuerdo a Panhuis et al. (Panhuis, 2003) el complejo Triton-X 100 tratado con CNTs interactuó eficazmente con la superficie hidrofilica de una enzima.

Por otra parte, uno de los métodos altamente preferidos en el desarrollo de inmunosensores es la adición de moléculas bifuncionales como el éster del ácido succinimidil I-pirenebutanoico o el complejo Tween 20 (Chen, 200I). Dicho éster puede adsorberse irreversiblemente en medio orgánicos (e.g., metanol) a la superficie hidrofóbica del nanotubo por medio de interacciones del tipo $\pi$. El resto del éster reacciona con las aminas primarias o secundarias que existen en la superficie de los anticuerpos por medio de sustituciones nucleofílicas. Otras moléculas que pueden dispersar a los CNTs en agua por medio de interacciones no covalentes son la amilasa (Kim, 2003), goma arábica (Bandyopadhyaya, 2002), ciclo dextrinas (Chen, 200I; Dodziuk, 2003), Nafion (Wang, 2003), entre otras. Finalmente, una vez conseguida la solubilización de los CNTs es necesario diseñar estrategias para incorporar funciones de reconocimiento molecular específico sobre estos nanoagregados; la forma más fácil y viable para reconocer moléculas específicas es mediante la adsorción de un antígeno o una enzima sobre el cuerpo del CNTs.

Un esquema simple para realizar un enlazamiento no específico sobre el cuerpo de un CNT es mediante el empleo de proteínas y ADN (Figura 5). Esta interacción ocurre preferencialmente por la vía no covalente $y$ ha sido especialmente observada con nanotubos oxidados (Kam, 2005).Además, debido a que la adsorción no covalente de anticuerpos sobre la superficie de los CNTs carece de un previo tratamiento químico riguroso, como los realizados por funcionalizaciones covalentes, la posibilidad de pérdida de habilidad por capturar al antígeno es pequeña; por ejemplo, estreptavidin, proteína $A$, albúmina de suero bovino y cyt c fueron adsorbidos espontáneamente a CNTs oxidados aunque no presentaron un patrón específico de enlazamiento. Las proteínas entonces fueron fácilmente transportadas por los CNTs al interior de varias células de mamíferos vía la ruta de endocitosis. Una vez ingresado en el citoplasma el complejo proteína-CNT desarrolló sus funciones biológicas, lo último evidenciado por la inducción de apoptosis por el transporte de cyt $c$.
Diferentes ensayos de inmune detección cuantitativos también han sido llevados a cabo. Por ejemplo, un método para detectar la cantidad de anticuerpos adsorbidos por CNTs ha sido desarrollado por O'Connor y colaboradores (O'Connor, 2004). El método consiste en incubar a $\mathrm{pH}$ fisiológico y por 3 horas un anticuerpo (e.g., antibiotina) junto con CNTs depositados previamente sobre grafito. Así se logró cuantificar que por cada I ml de solución de anticuerpo se saturan $0,5 \mathrm{mg}$ CNTs. Por otra parte, dos mecanismos de transducción diferentes fueron ensayados por Chen (Chen, 200I) con el fin de medir la sensibilidad de la detección de anticuerpos. Uno de ellos consistió en formar una película ultra delgada de CNTs sobre una superficie de un cristal de cuarzo y los cambios en masa fueron medidos por micro balanza igualmente de cuarzo. El segundo involucró el desarrollo de un transistor formado por un nanotubo (como puente) y dos electrones de Ti/Au. El antígeno, un factor de empalme UIA ARN, fue conjugado con Tween 20 el cual a la vez se adsorbe fácilmente al cuerpo de los CNTs. La detección de un auto anticuerpo específico para ambos sistemas fue menor a I nM.

No obstante, muchas veces el tamaño promedio de ciertas proteínas como las inmunoglobulinas (7 $8 \mathrm{~nm}$, IgG humana, $170 \mathrm{kDa}$ ) puede ser un factor que afecte la estabilidad del enlazamiento (los diámetros de CNTs varían entre I - $2 \mathrm{~nm})(\mathrm{Kam}, 2005)$. Debido a este impedimento se ha encontrado (Chen, $200 \mathrm{lb}$ ) que ferritin no es capaz de adsorberse directamente sobre CNTs; en el caso de proteínas como las peroxidadas o quimotripsina se encontró que los valores están alrededor de 0,5 - 0,6 mg de proteína por cada mg de CNTs (Karajanagi, 2004). Así, la cantidad de anticuerpos adsorbidos por unidad de peso de nanotubos es uno de los parámetros más críticos para el desarrollo de biosensores.

\subsection{Conductores eléctricos}

Como se ha mencionado anteriormente, las propiedades eléctricas de los CNTs (e.g., conductividad) conceden a estos nanomateriales características únicas. CNTs han sido empleados como electrodos a fin de mediar en reacciones de transferencia de carga en un medio acuoso con especies electroactivas (Britto, 1996; Campbell, 1999; Chen, 2003; Luo, 200 I; Wang, 2003b). Electrodos de CNTs fueron empleados para examinar la oxidación de dopamina a dopaminaquinona, la cual demostró una reversibilidad ideal en voltametría cíclica y fue significantemente superior a las observadas en otros electrodos de carbono (Britto, 1996). 


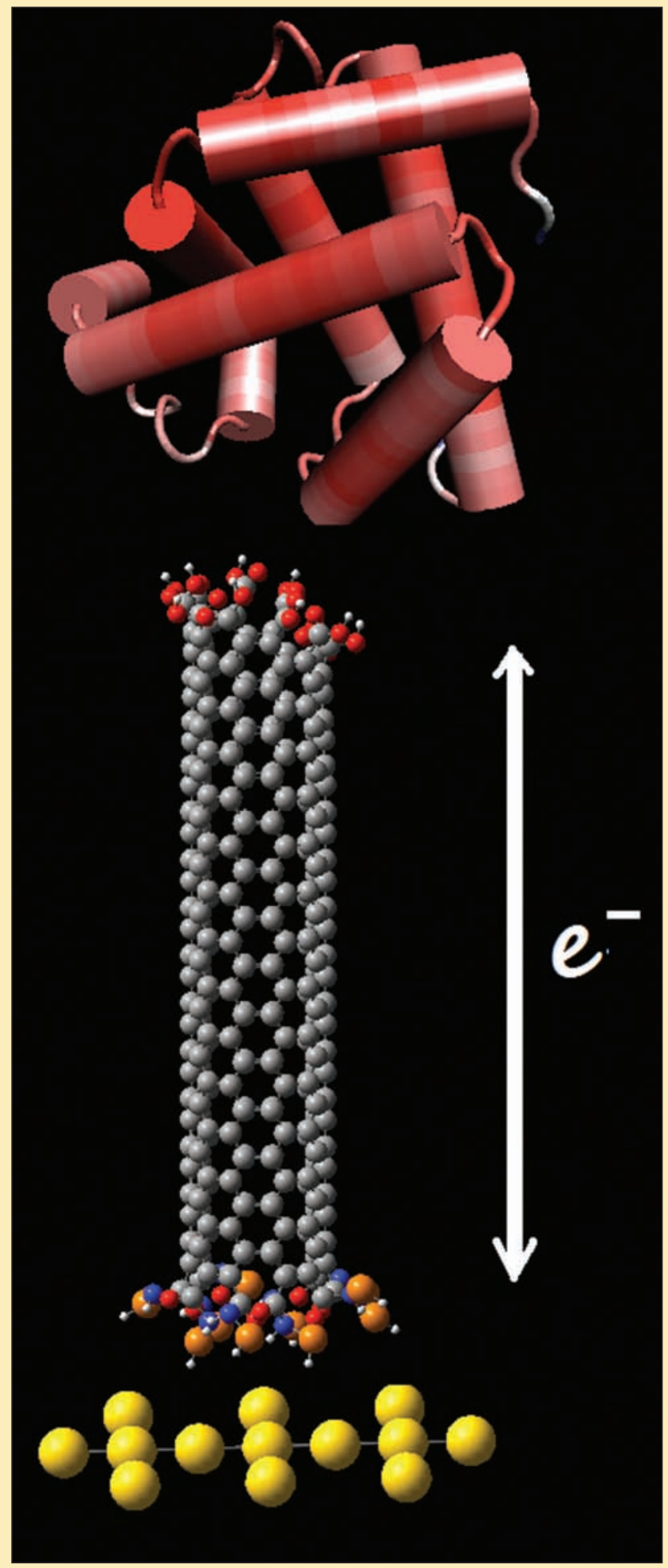

Figura 6. Representación gráfica de la base para el desarrollo de un electrodo empleado en la inmovilización y transporte de carga con biomoléculas. El electrodo está conformado por un ensamble ordenado de CNTs alineados verticalmente sobre una superficie de átomos de Au. Obsérvese como las puntas derivatizadas del nanotubo interactúan vía covalente con la superficie metálica (i.e. - $\mathrm{CO}-\mathrm{NH}-\mathrm{S}-\mathrm{Au}$ ) y vía no covalente con la biomolécula (e.g. enzimas oxidoreductasas) permitiendo así el flujo de carga entre ambos sistemas. Fuente: Flavio F. Contreras-Torres.
En términos generales, la construcción de un electrodo de nanotubos de carbono es muy simple y básicamente consiste en formar una pasta de CNTs (ca. $10 \mathrm{mg}$ ) con bromoformo (ca. $10 \mu \mathrm{l})$. La pasta posteriormente se introduce en un pequeño tubo de vidrio de aproximadamente $8 \mathrm{~cm}$ de largo y $3-5 \mathrm{~mm}$ de diámetro. Entonces, en un extremo del tubo que contiene la pasta de CNTs se inserta un alambre de platino ( $\mathrm{Pt}$ ), (aunque también se ha empleado cobre) y su extremo libre se sujeta al tubo de vidrio con cinta de embalaje. Sin embargo, otras alternativas para preparar microelectrodos de fibras CNTs también se han desarrollado (Wang, 2003b). Las fibras de CNTs (ver Vigolo, 2000) son preparadas por un proceso de hilado que involucra la inyección de una suspensión acuosa concentrada de CNTs a un baño para su coagulación. Este proceso de preparación da como resultado una novedosa forma de obtener redes interconectadas de CNT con diversos polímeros. Las fibras de CNTs son posteriormente activadas a $300{ }^{\circ} \mathrm{C}$ por I h. Las ventajas de estos electrodos tipo fibras ha sido demostrada en reacciones electrocatalíticas con importantes biomoléculas como el NADH o la dopamina.

Por otra parte, los últimos avances en la producción de arreglos ordenadamente alineados de CNTs han demostrado ser otra ruta para el desarrollo de la nanotecnología de biosensores (Chattopadhyay, 200I; Liu, 2000; Wu, 200I). Para este caso, el ensamble orientado de CNTs cortos normalmente viene acompañado de un ataque covalente sobre los extremos del nanotubo y donde uno de los extremos está adherido a una superficie que presenta partículas metálicas. De esta forma el arreglo permite transportar carga electrónica desde un extremo al otro, formando un lugar donde se pueden adherir biomoléculas (Figura 6).

\subsection{Transistores de efecto de campo}

De acuerdo a la electrónica molecular la tecnología de los transistores nanoarquitecturados podría desarrollarse también a partir CNTs (Avouris, 2002; Bradley, 2003; Javey, 2004). En general, la base de los transistores de efecto de campo (FET, por sus siglas en inglés) son dispositivos de conmutación de tres terminales que consisten en un semiconductor conectado a dos electrodos metálicos. Mediante la aplicación de un voltaje a uno de los electrodos, el material semiconductor puede pasar a un estado aislante. Dado las propiedades conductoras de los materiales, muchos de los FET han sido desarrollados para trabajar a bajas temperaturas. No obstante, Tans y colaboradores 


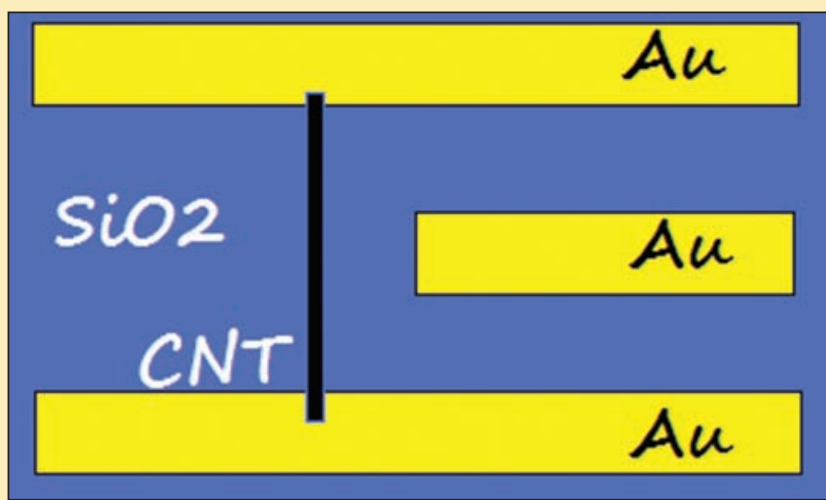

Figura 7. Representación gráfica de un transistor de efecto de campo (FET, por sus siglas en inglés) donde la nanotecnología ha incorporado un nanotubo de carbono entre los dos electrodos de oro.

(Dekker, 1998) han desarrollado FETs que trabajan a temperatura ambiente, a partir de, CNTs metálicos. Basados en este diseño otras investigaciones también se han enfocado al desarrollo de nanosensores reemplazando al electrodo de estado sólido por moléculas que modulan la conductancia en el nanotubo (Bradley, 2003b; Collins, 2000; Kong, 2000; Someya, 2003; Star, 2003, 2004).

Recientes investigaciones (Bradley, 2004; Star, 2003b) han demostrado, a través de los FET basados en CNTs (CNTFET, para acortar), la capacidad de detección de interacciones antígeno-anticuerpo. En particular, enlaces no específicos para el par Biotinia-Estreptadivina fueron detectados y donde la biotina previamente fue adherida al cuerpo del nanotubo con una capa del copolímero PEI/PEG. El proceso de enlazamiento del anticuerpo al CNT funcionalizado con el antígeno fue detectado por análisis microgravimétricos. Por otra parte, la detección de cyt $c$ vía los cambios en las propiedades de transporte electrónico de un transistor CNTFET también ha sido reportada (Boussaad, 2003).

Finalmente, nanotransistores basados en nanocircuitos grabados de ADN (Keren, 2002 \& 2003) han sido desarrollados para la detección de proteínas (Figura 7). La conexión precisa de los nanoelectrodos de oro (Au) con un CNT fue realizada por medio de un ensamble bioquímico. Los artefactos exhiben incrementos característicos en la relación corriente vs. voltaje.

\section{Conclusiones}

Con el advenimiento de la nanotecnología muchos cambios fundamentales en la forma de concebir al mundo estarán pronto por presenciarse. Biotecnología y ciencia de materiales son los campos que estarán más comprometidos con dicho desarrollo tecnológico. Un camino, sin límites claros, está por descubrirse abordando las preguntas no resueltas y captando todas las oportunidades que nos ofrecen los nanotubos de carbono como trasportadores de carga y soportes moleculares. La presente revisión se ha enfatizado principalmente en el uso de nanomateriales biohíbridos para usos biomédicos, por ejemplo, dada la incorporación de ADN con nanotubos de carbono se puede abrir la posibilidad para el desarrollo de biosensores específicos. Se ha demostrado cómo el desarrollo de contactos eléctricos directos por medio de nanotubos de carbono entre enzimas como las reductasas y electrodos macroscópicos representa el puente de enlace entre la tecnología de los bisensores y la nanotecnología. Dichos novedosos sistemas biohíbridos enzimas/CNTs podrían permitir la generación de sorprendentes aplicaciones tecnológicas como nanoceldas de biocombustibles. Las perspectivas actuales para el desarrollo de nanosistemas biohíbridos son alentadoras, sin embargo, la integración de CNTs con otros materiales electrónicos como polímeros conductores o nanopartículas metálicas es también un área de estudio con prometedores avances tecnológicos. Fundados en los avances presentados, tanto los estudios como el desarrollo de aplicaciones en nanobiotecnología se anticipa como una nueva rama de las ciencias inter disciplinarias.

\section{Agradecimientos}

F. F. C.-T. agradece a la Dirección General de Personal Académico DGAPA y a la Universidad Nacional Autónoma de México por los aportes económicos. El autor reconoce de manera especial tanto al Prof. Dr. Vladimir A. Basiuk (Instituto de Ciencias Nucleares, UNAM) como a la Dra. Elena Golovataya Dhzymbeeva (Centro de Ciencias Aplicadas y Desarrollo Tecnológico, UNAM) por su apoyo moral y técnico en el desarrollo de nuevas técnicas experimentales por microscopía de fuerza atómica para el estudio de biomoléculas.

\section{Referencias}

Avouris, P. 2002. Molecular electronics with carbon nanotubes. Acc. Chem. Res. 35, I026-1034.

Balasubramanian, K. and Burghard, M. 2006. Biosensors based on carbon nanotubes. Anal. Bioanal. Chem. $385,452-468$. 
Bandyopadhyaya, R., E. Nativ-Roth, O. Regev and R. Yerushalmi-Rozen. 2002. Stabilization of individual carbon nanotubes in aqueous solutions. Nano Lett. 2, 25-28.

Barik, T. K., B. Sahu and V. Swain. 2008. Nanosilica - from medicine to pest control. Parasitol. Res. I03, 253258.

Beaux II, M. F., D. N. Mcllroy and K. E. Gustin. 2008. Utilization of solid nanomaterials for drug delivery. Expert Opin. Drug Delivery 5, 725-735.

Binning, G., H. Rohrer, Ch. Gerber and E.Weibel. 1982. Surface studies by scanning tunneling microscopy. Phys. Rev. Lett. 49, 57-6I.

Binnig, G., C. F. Quate and Ch. Gerber. 1986. Atomic force microscope. Phys. Rev. Lett. 56, 930-933.

Boussaad, S., N. J. Tao, R. Zhang, T. Hopson and L. A. Nagahara. 2003. In situ detection of cytochrome c adsorption with single walled carbon nanotube device. Chem. Commun. I3, I502-I 503.

Bradley, K., J.-C. P. Gabriel and G. Grüner. 2003. Flexible nanotube electronics. Nano Lett. 3, I 353-I 355.

Bradley, K., J. Cumings, A. Star, J.-C. P. Gabriel and G. Grüner. 2003b. Influence of mobile ion on nanotube based FET devices. Nano Lett. 3, 639-64I.

Bradley, K., M. Briman, A. Star, G. Grüner. 2004. Charge transfer from adsorbed proteins. Nano Lett. 4, 253-256.

Britto, P.J ., K. S.V. Santhanam and P. M. Ajayan. 200I. Carbon nanotube electrode for oxidation of dopamine. Bioelectrochem. Bioenerg. 4I, I2I - I 25.

Campbell, J. K., L. Sun and R. M. Crooks. 1999. Electrochemistry using carbon nanotubes. J.Am. Chem. Soc. I 2 I, 3779-3780.

Collins, P. G., K. Bradley, M. Ishigami and A. Zettl. 2000. Extreme oxygen sensitivity of electronic properties of carbon nanotubes. Science 287, I80 I1804.

Contreras-Torres, F. F., and V. A. Basiuk. ONIOM studies of esterification at oxidized carbon nanotubes tips. J. Phys.: Conf. Series 6 I, 85-89.

Contreras-Torres, F. F., O. Amelines-Sarria, A. F. Jalbout, E.V. Basiuk and V. A. Basiuk. 2008. Interaction between cation-encapsulated single-walled carbon nanotubes $\mathrm{M}^{+} @ S W N T\left(\mathrm{M}^{+}=\mathrm{H}, \mathrm{Li}, \mathrm{Na}\right)$ and nucleophiles. Comput. Mater. Sci. 44, 240-246.

Contreras-Torres, F. F., A. F. Jalbout, O. F. Amelines and V. A. Basiuk. 2008b. Interaction between cation-encapsulated single-walled carbon nanotubes with small polar molecules. J. Phys. Chem. C I I 2, 2736-2742.

Contreras-Torres, F. F., A. Arzate-Jacinto and V. A. Basiuk. 2008c. Direct esterification of oxidized defects in single-walled carbon nanotubes tips: a comparison of several ONIOM computational schemes. J. Comput. Theor. Nanosci. 5, 93-I0I.

Chattopadhyay, D., I. Galeska and F. Papadimitrakopoulos. 200I. Metal-assisted organization of shortened carbon nanotubes in monolayer and multilayer forest assemblies. J. Am. Chem. Soc. I 23, 945I-9452.

Chen, C. S., M. Mrksich, S. Huang, G. M. Whitesides and D. E. Ingber. 1997. Geometric control of cell life and death. Science 276, I425- I 428.

Chen, J., M. J. Dyer, and M. F.Yu. 200I. Cyclodextrin-mediated soft cutting of single-walled carbon nanotubes. J. Am. Chem. Soc. I 23, 6201-6202.

Chen, R. J.,Y. Zhang, D.Wang and H. Dai. 200 Ib. Noncovalent sidewall functionalization of single-walled carbon nanotubes for protein immobilization. J. Am. Chem. Soc I 23, 3838-3839.

Chen, R.-S., W.-H. Huang, H. Tong, Z.-L. Wang and J.-K. Cheng. 2003. Carbon fiber nanoelectrodes modified by single-walled carbon nanotubes. Anal. Chem. 75, 634I-6345.

Delgado, G. C. 2008. Guerra por lo invisible: negocio, implicaciones y riesgos de la nanotecnología. Ceiich, UNAM. México.

Davis, J. J., M. L. H. Green, H. A. O. Hill,Y. C. Leung, P. J. Sadler, J. Sloan, A.V. Xavier and S. C. Tang. 1998. The immobilization of proteins to carbon nanotubes. Inorg. Chim. Acta 272, 26I-266.

Dinu, C. Z., S. S. Bale, D. B. Chrisey and J. S. Dordick. 2009. Manipulation of individual carbon nanotubes by reconstructing the intracellular transport of a living cell. Adv. Mater. 20, I-5.

Dodziuk, H., A. Ejchart, W. Anczewski, H. Ueda, E. Krinichnaya, G. Dolgonos and W. Kutner. 2003. Water solubilization, determination of the number of different types of single-wall carbon nanotubes and their partial separation with respect to diameters by complexation with beta-cyclodextrin. Chem. Commun. (Cambridge) 8, 986987.

Drexler, K. E. 1986. Engines of creation:The coming era of nanotechnology. New York, Anchor Press.

Drexler, K. E. 1991. Molecular machinery and manufacturing with applications to computation. PhD Thesis, Massachusetts Institute of Technology.

Dulinska, I., M. Targosz, W. Strojny, M. Lekka, P. Czuba,W. Balwierz and M. Szymonski. 2006. Stiffness of nor$\mathrm{mal}$ and pathological erythrocytes studied by means of atomic force microscopy. J. Biochem. Biophys. Methods 66, I-II.

Eschenbach, von A. C. 2007. Nanotechnology: a report of the U.S. Food and Drug Administration. Nanotechnology Task Force, July, 25. Disponible como report2007.pdf en www.fda.gov/nanotechnology/taskforce.

Gen, Y., P. Dalhaimer, S. Cai, R. Tsai, M. Tewari, T. Minko and D. E. Discher. 2007. Shape effects of filaments versus spherical particles in flow and drug delivery. Nature Nanotech. 2, 249-255.

Gooding, J. J., R.Wibowo, J. Liu, W. Yang, D. Losic, S. Orbons, F.J. Mearns, J. G. Shapter and D. B. Hibbert. 2003. Protein electrochemistry using aligned carbon nanotube arrays. J.Am. Chem. Soc. I25, 9006-9007. 
Griffith, L. G. and M.A. Swartz. 2006. Capturing complex 3D tissue physiology in vitro. Nature Rev. Mol. Cell. Biol. 7, 2 II-224.

Harutyuyan, A. R. B. K. Pradhan, G. U. Sumanasekera, E. Yu. Korobko and A. A. Kuznetsov. (2002). Carbon nanotubes for medical applications. European Cell. Mater. 3, 84-87.

Hussain, S. M., L. K. Braydich-Stolle, A. M. Schrand, R. C. Murdock, K. O.Yu, D. M. Mattie, J. J. Schlager and M. Terrones. 2009. Toxicity evaluation for safe use of nanomaterials: recent achievements and technical challenges. Adv. Mater. 2 I, I-I I.

lijima, S. 1991. Helical microtubules of graphitic carbon. Nature 354, 56-58.

Javey, A., J. Guo, D. B. Farmer, Q.Wang, D.Wang, R. G. Gordon, M. Lundstrom and H. Dai. 2004. Carbon nanotube field-effect transistors with integrated ohmic contacts and high-? gate dielectrics. Nano Lett. 4, 447-450.

Joy, B. 2000. Why the future doesn't need us. Wired Magazine 8.

Kam, N.W.S. and H. Dai. 2005. Carbon nanotubes as intracellular protein transporters: generality and biological functionality. J. Am. Chem. Soc. | 27, 602|-6026.

Katz, E. and I. Willner. 2004. Biomolecule-functionalized carbon nanotubes: applications in nanobioelectronics. Chem. Phys. Chem. 5, I084-I 04.

Karajanagi, S. S., A. A. Vertegel, R. S. Kane and J. S. Dordick. 2004. Structure and function of enzymes adsorbed onto single-walled carbon nanotubes. Langmuir 20, II594- I I599.

Keren, K., M. Krueger, R. Gilad, G. Ben-Yoseph, U. Sivan and E. Braun. 2002. Sequence-specific molecular lithography on single DNA molecules. Science 297, 72-75.

Keren, K., R. S. Berman, E. Buchstab, U. Sivan and E. Braun. 2003. DNA-template carbon nanotube fieldeffect transistor. Science 302, I380-1382.

Kienberger, F., A. Ebner, H. J. Gruber and P. Hinterdorfer. 2006. Molecular recognition imaging and force spectroscopy of single biomolecules. Acc. Chem. Res. 39, 29-36.

Kim, O. K., J. Je, J.W. Baldwin, S. Kooi, P. E. Pehrssona and L. J. Buckley. 2003. Solubilization of single-wall carbon nanotubes by supramolecular encapsulation of helical amylose.J.Am. Chem. Soc. I 25, 4426-4427.

Kong, J., N. R. Franklin, C.W. Zhou, M. G. Chapline, S. Peng, K. Cho and H. J. Dai. 2000. Nanotube molecular wires as chemical sensors. Science 287, 622-625.

Kong, J., M. G. Chapline and H. Dai. 200I. Functionalized carbon nanotubes for molecular hydrogen sensors. Adv. Mater. I 3, I 384- I 386.

Kroto, H.W., J. R. Heath, S. C. O'Brien, R. F. Curl and R. E. Smalley. 1985. $\mathbf{C}_{\mathbf{6 0}}$ : buckminsterfullerene. Nature $318,162-163$.

Langer, R. and D.A.Tirrell. 2004. Designing materials for biology and medicine. Nature 428, 487-492.

Li, Q., J. Zhang, H. Yan, M. He and Z. Liu, Z. 2004. Thionine-mediated chemistry of carbon nanotubes. Carbon 42, 287-291.

Li, G., J. Liao, G. Hu, N. Ma and P.Wu. 2005. Study of carbon nanotube modified biosensor for monitoring total cholesterol in blood. Biosens. Bioelectron. 20, 2| 40-2। 44.

Liang, Z., R. Lao, J. Wang, Y. Liu, L. Wang, Q. Huang, S. Song, G. Li and C. Fan. 2007. Solubilization of singlewalled carbon nanotubes with singlestranded DNA generated from asymmetric PCR. Int. J.Mol. Sci. 8, 705-7। 3.

Linazasoro, G. 2008. Potential applications of nanotechnologies to Parkinson's disease therapy. Parkinsonism Related Disorders 14, 383-392.

Liu, Z., Z. Shen.T. Zhu, S. Hou, L.Ying, Z. Shi and Z. Gu. 2000. Organizing single-walled carbon nanotubes on gold using a wet chemical self-assembling technique. Langmuir 16, 3569-3573.

Luo, H., Z. Shi, N. Li, Z. Gu and Q. Zhuang. 200I. Investigation of the electrochemical and electrocatalytic behavior of single-walled carbon nanotube film on a glassy carbon electrode. Anal. Chem. 73, 915-920.

Mashelkar, R. A. 2006. Bionanotechnology: proteins to nanodevices. Springer.

Mitragotri S. and J. Lahann. 2009. Physical approaches to biomaterial design. Nature Mater. 8, 15-23.

Monthioux, M. and V. L. Kuznetsov. 2006. Who should be given the credit for the discovery of carbon nanotubes? CARBON 44, I62I-1625.

Mooney, D. J. and A. G. Mikos. 1999. Growing new organs. Sci. Am. 280, 60-65.

Moore, V. C., M. S. Strano, E. H. Haroz, R. H. Hauge, and R. E. Smalley. 2003. Individually suspended singlewalled carbon nanotubes in various surfactants. Nano Lett. 3, I379-I 382.

Morgan, C., C. Newman and C. Price. 1996. Immunosensors: technology and opportunities in laboratory medicine. Clin. Chem. 42, 193-209.

Muro, S. 2008. Control of endothelial targeting and intracellular delivery of therapeutic enzymes by modulating the size and the shape of ICAM-I-targeted carriers. Mol. Ther. I6, I4501458.

O'Connor, M., S. N. Kim, A. J. Killard, R. J. Forster, M. R. Smyth, F. Papadimitrakopoulos and J. F. Rusling. 2004. Mediated amperometric immunosensing using single walled carbon nanotubes forests. Analyst I29, I I76-। I80.

Panhuis, M. i. h.; C. Salvador, E. Franklin, G. Chambers, A. Fonseca, J. B. Nagy, W. J. Blau and A. I. Minett. 2003. Characterization of specific interaction between functionalized carbon nanotubes and an enzyme. J. Nanosci. Nanotechnol. 3, 209-2I 3.

Ralston, J., I. Larson, M.W. Rutland,A.A. Feiler and M. Kleijn. 2005. Atomic force microscopy and direct 
surface force measurements (IUPAC technical report). Pure Appl. Chem. 77, 2I49-2I 70.

Roos, W. H. and G. J. L.Wuite. 2009. Nanoidentation studies reveal material properties of viruses. $A d v$. Mater. 2 I, I-6.

Sifferd, K. L. 2008. Nanotechnology and the attribution of responsibility. Nanotech. Law \& Business. 5, 177-189.

Shie, J.W., U. Yogeswaran and S. M. Chen. 2008. Electroanalytical properties of cytochrome c by direct electrochemistry on multi-walled carbon nanotubes incorporated with DNA biocomposite film. Talanta 74, I659-1669.

Someya, T., J. Small, P. Kim, C. Nuckolls and J.T.Yardley. 2003. Alcohol vapor sensors based on single-walled carbon nanotube field effect transistors. Nano Lett. 3, 877-88I.

Star, A., T. R. Han, J.-C. P. Gabriel, K. Bradley and G. Grüner. 2003. Interaction of aromatic compounds with carbon nanotubes: Correlation to the hammett parameter of the substituent and measured carbon nanotube FET response. Nano Lett. 3, I42|-| 423.

Star, A., J.-C. P. Gabriel, K. Bradley and G. Grüner. 2003b. Electronic detection of specific protein binding using nanotube FET devices. Nano Lett. 3, 459-463.

Star, A., T.-R. Han, V. Joshi and J. R. Stetter. 2004. Sensing with Nafion coated carbon nanotube field-effect transistors. Electroanalysis 16, 108-II2.

Strand R. y R. Nydal. 2008. Nanoética buena - Nanotecnología buena. Mundo Nano I, 6I-79.

Sun, Y. and H. H. Wang. 2007. High-performance, flexible hydrogen sensors that use carbon nanotubes decorated with palladium nanoparticles. Adv. Mater. 19, 28I8-2823.

Tang, H., J. Chen, S. Yao, L. Nie, G. Deng and Y. Kuang. 2004. Amperometric glucose biosensor based on adsorption of glucose oxidase at platinum nanoparticle-modified carbon nanotube electrode. Anal. Biochem. 331, 89-97.

Tans, S. J., R. M. Verschueren and C. Dekker. 1998. Roomtemperature transistor based on a single carbon nanotube. Nature 393, 49-52.

Teles, F. R. R. and L. P Fonseca. 2008. Trends in DNA biosensors. Talanta 77, 606-623.

The NanoBusiness Alliance. 200I. 200 I Business of nanotechnology survey. p. 12.

Veetil, J.V. and K. Ye. 2007. Develompment of immunosensors using carbon nanotubes. Biotechnol. Prog. 23, 5I7-531.

Vestergaard, M. K. Kerman and E.Tamiya, E. 2007. An overview of label-free electrochemical protein sensors. Sensors 7, 3442-3458.

Vigolo, B., A. Penicaud, C. Coulon, C. Sauder, R. Pailler, C. Journet, P. Bernier, P. Poulin. 2000. Macroscopic fibers and ribbons of oriented carbon nanotubes. Science 290, I33|-1334.
Wang, J., M. Musameh and Y. Lin. 2003. Solubilization of carbon nanotubes by Nafion toward the preparation of amperometric biosensors. J. Am. Chem. Soc. I 25, 2408-2409.

Wang, J., R. P. Deo, P. Poulin and M. Mangey. 2003b. Carbon nanotube fiber microelectrodes. J. Am. Chem. Soc. I 25, I 4706- I 4707.

Wu, B., J. Zhang, Z. Wei, S. Cai and Z. Liu. 200I. Chemical alignment of oxidatively shortened single-walled carbon nanotubes on silver surface. J. Phys. Chem. B I 05, 5075-5078.

Yogeswaran, U. and S. M. Chen. 2007. Separation and concentration effect of $f$-MWCNTs on electrocatalytic responses of ascorbic acid, dopamine and uric acid at $f$-MWCNTs incorporated with poly (neutral red) composite films. Electrochim. Acta. 52, 5985-5996.

Yogeswaran, U. S. Thiagarajan and S. M. Chen. 2007. Pinecone shape hydroxypropyl-,-cyclodextrin on a film of multi-walled carbon nanotubes coated with gold particles for the simultaneous determination of tyrosine, guanine, adenine and thymine. Carbon 45, 2783-2796.

Yogeswaran, U., S. Thiagarajan and S. M. Chen. 2007b. Nanocomposite of functionalized multiwall carbon nanotubes with nafion, nano platinum, and nano gold biosensing film for simultaneous determination of ascorbic acid, epinephrine, and uric acid. Anal. Biochem. 365, I22I3I.

Yogeswaran, U., S. Thiagarajan, and S. M. Chen. 2007c. Pinecone shape hydroxypropyl-,-cyclodextrin on a film of multi-walled carbon nanotubes coated with gold particles for the simultaneous determination of tyrosine, guanine, adenine and thymine. Carbon 45, 2783-2796.

Yogeswaran, U. and S. M. Chen. 2008. A Review on the electrochemical sensors and biosensors composed of nanowires as sensing material. Sensors 8, 290-313.

Yogeswaran, U. and S. M. Chen. 2008b. Multi-walled carbon nanotubes with poly(methylene blue) composite film for the enhancement and separation of electroanalytical responses of catecholamine and ascorbic acid. Sens. Actuat. B: Chem. I 30, 739-749.

Zhang, J., J. K. Lee, Y.Wu and R.W. Murray. 2003. Photoluminescence and electronic interaction of anthracene derivatives adsorbed on sidewalls of single-walled carbon nanotubes. Nano Lett. 3, 403-407.

Zhou, B., R. Sun, X. Hu, L.Wang, H.Wu, S. Song and C. Fan. 2005. Facile interfacial electron transfer of hemoglobin mediated by conjugated polymers. Int. J. Mol. Sci. 6, 303-310. 